\title{
SIMILARITY TRANSFORMATIONS OF HYPERSURFACES
}

\author{
YUEN-FAT WONG ${ }^{1}$
}

It is the purpose of this note to extend C. S. Hsü's theorem [4] to $n$ dimensions, $n \geqq 2$. In order to do that we make use of the computations of $[3$, pp. 89-90].

Notations. $M_{1}, M_{2}$ are $n$-dimensional closed orientable Riemannian manifolds of class $C^{3}$, imbedded in $n+1$ dimensional Euclidean space. $\Delta_{2}$ is the second differential operator of Beltrami. $\Delta_{1}$ is the operator defined by

$$
\Delta_{1} \sigma=g^{i j} \frac{\partial \sigma}{\partial x^{i}} \frac{\partial \sigma}{\partial x^{j}} .
$$

As usual, $g_{i j}$ is the positive-definite metric tensor of $M_{1}$ and $\left(g^{i j}\right)$ $=\left(g_{i j}\right)^{-1}, R=g^{i j} R_{i j}$, where $R_{i j}$ is the Ricci tensor. We also use repeated index for summation. We denote corresponding elements of $M_{2}$ by attaching accents.

Lemмa $[5, p .30]$. In a compact Riemannian manifold with positive-definite metric, if a function $\sigma$ satisfies

$$
\Delta_{2} \sigma \geqq 0
$$

everywhere in the manifold, then $\sigma$ is a constant.

Theorem. Given $M_{1}, M_{2}$ with positive $R, R^{\prime}$, respectively, and a diffeomorphism $h: M_{1} \rightarrow M_{2}$ which preserves $R I$. ( $I$ is the first fundamental form.) Then $h$ is a similarity.

Proof. It is sufficient to show that $h$ followed by a homothetic transformation is a rigid motion. We first show that $I^{\prime} / I$ is a constant. Let $I^{\prime} / I=R / R^{\prime}=e^{2 \sigma}$. Then $g_{i j}^{\prime}=e^{2 \sigma} g_{i j}$. By [3, pp. 89-90], we have

$$
\left(R+2(n-1) \Delta_{2} \sigma+(n-1)(n-2) \Delta_{1} \sigma\right)=R^{\prime} e^{2 \sigma} .
$$

Making use of the hypothesis, we obtain

$$
\Delta_{2} \sigma=-\{(n-2) / 2\} \Delta_{1} \sigma
$$

Since $\Delta_{1} \sigma \geqq 0$,

$$
-\Delta_{2} \sigma=\Delta_{2}(-\sigma)=\{(n-2) / 2\} \Delta_{1} \sigma \geqq 0 .
$$

Received by the editors January 10, 1963.

1 The author is indebted to C. S. Hsü for his suggestions. 
By the lemma, $\sigma$ is a constant. Consequently, $I^{\prime} / I$ is also a constant. Now we divide the proof into two different cases.

Case 1. For $n=2$, the fact that $h$ followed by a homothetic transformation of a proportionality constant $\left(R^{\prime} / R\right)^{-1 / 2}$ is the desired rigid motion follows from Cohn-Vossen's theorem.

Case 2. For $n>2$, the above fact follows from a different argument (cf. [1, pp. 26-27]). It says that isometric hypersurfaces in Euclidean space of dimension greater than three are congruent or symmetric.

\section{REFERENCES}

1. S. S. Chern, Topics in differential geometry, Mimeographed notes, Institute for Advanced Study, Princeton, N. J., 1951.

2. S. Cohn-Vossen, Zwei Sätze über die Starrheit der Einflächen, Nachr. Ges. Wiss. Gott. (1927), 125.

3. L. P. Eisenhart, Riemannian geometry, Princeton Univ. Press, Princeton, N. J., 1926.

4. C. S. Hsü, Generalization of Cohn-Vossen's theorem, Proc. Amer. Math. Soc. 11 (1960), 845-846.

5. K. Yano and S. Bochner, Curvature and Betti numbers, Annals of Mathematics Studies No. 32, Princeton Univ. Press, Princeton, N. J., 1953.

Cornell University 